\title{
Neutrino oscillation at accelerators
}

\author{
Klaus Winter \\ CERN, Geneva
}

Invited talk presented at the meeting on

"Neutrino Astronomy"

at The Royal Society, London, on 9 and 10 June 1993

\begin{abstract}
$\underline{\text { Abstract }}$
From measurements of the width and of the peak value of the $Z^{\circ}$ pole in $\mathrm{e}^{+} \mathrm{e}^{-}$annihilations the existence of three neutrino families in nature has recently been derived. Do these families mix or are they distinct? We do not know if the mixing phenomenon exists in nature. Two different types of experiments have been conducted at high energy accelerators in searching for the resulting neutrino oscillation, the apparent disappearance of neutrinos of one flavour as they are propagating and the appearance of neutrinos of a new flavour. Present limits, a new result, advantages and weaknesses of the two approaches and future prospects will be presented and discussed.
\end{abstract}




\section{INTRODUCTION}

From measurements of the width and of the peak value of the cross section of $\mathrm{e}^{+} \mathrm{e}^{-}$ annihilation to all visible final states at the $Z^{\circ}$ pole we know that there exist three families of light neutrinos in nature. Two of them have been detected by their capture on nucleons, the electron- and the muon-neutrino; the third, tau-neutrino, has remained undetected. Do these neutrinos have non-zero mass? Do these families mix or are they distinct? Non-zero neutrino mass is a necessary condition for neutrino mixing. These questions belong to the most fundamental ones for experimental particle physics. Answers may come from searches for neutrino oscillation. The mixing of neutrino families can be described by a matrix, similar to the Cabibbo-Kobayashi-Maskawa quark mixing matrix. Assuming neutrino masses are generated by a see-saw mechanism, oscillation will be dominantly a phenomenon between the families of the two heaviest leptons, the muon and the tau. The frequency of the oscillation term is given by the difference of the neutrino masses squared. The probability of appearance of neutrinos of a new flavour, $v_{b}$ in a beam of $v_{a}$ or disappearance of $v_{a}$ is given by the well-known expression :

$$
P_{a b}=\sin ^{2} 2 \Theta_{a b} \cdot \sin ^{2}\left(1.27 \frac{L}{E} \Delta m_{a b}^{2}\right)
$$

where $\mathrm{L}$ is the distance to the neutrino source in $\mathrm{Km}$ and $\mathrm{E}$ the neutrino energy in $\mathrm{GeV}$. Results from recent experiments on solar neutrinos, which are reported elsewhere at this meeting, are consistent with a Mikheyev-Smirnov-Wolfenstein solution to the solar neutrino problem [1]. One of the favoured solutions gives a mass to the $v_{\tau}$ which would make it a candidate for dark matter $\left(\mathrm{mv}_{\tau} \sim 20 \mathrm{eV}\right)$ [2]. Also recent experimental results on the unisotropy of the cosmic background radiation favour $v_{\tau}$ with $\mathrm{m}_{\tau} \sim 7 \mathrm{eV}$. Search for neutrino oscillation is straight forward at high energy accelerators. They are sources of collimated $v_{\mu}\left(\nabla_{\mu}\right)$ beams of high intensity. Their energy and the distance to the source are well-known and matched to detect oscillation for $m v_{\tau}>1 \mathrm{eV}$. Questions about the relative abundance of $v_{\mu}$ and $v_{e}$ were asked in the first neutrino experiment at CERN which confirmed the discovery of the muon-neutrino. It also detected a small component of 
electron-neutrinos. What was their origin? Were they produced by neutrino oscillation? Their abundance agreed with $\mathrm{K}_{\mathrm{e}}$ decays of kaons.

\section{EXPERIMENTAL METHODS}

Two methods have been used for this search. One consists in searching for the appearance of a neutrino of another family $\left(v_{b}\right)$ difference from the dominant component $\left(v_{a}\right)$ of the beam, e.g. $v_{\tau}$ or $v_{\mathrm{e}}$. The other method consists of searching for the corresponding disappearance of $v_{\mathrm{a}}$. The relation of the two methods is given by unitarity

$$
\mathrm{P}_{\mathrm{aa}}=1 \cdot \mathrm{P}_{\mathrm{ab}} \text {. }
$$

Both methods have distinct advantages and disadvantages.

\subsection{Disappearance experiments}

The disappearance search is inclusive; $v_{a}$ may disappear to any kind of neutrino. The sensitivity to different values of the $\Delta \mathrm{m}^{2}$ can be extended to smaller values by increasing the distance of the detector to the source. The number of events $\mathrm{N}$ induced by $\mathrm{v}_{\mathrm{a}}$ in this FAR detector can be firmly predicted by measuring simultaneously their number in a NEAR detector, provided the beam can be accurately described as coming from a point source. In this case

$$
R=\frac{N(\text { FAR })}{N(\text { NEAR })}=\frac{M(\text { FAR }) / S(\text { FAR })}{M(\text { NEAR }) / S(\text { NEAR })}\left(\frac{L(\text { NEAR })}{L(\text { FAR })}\right)^{2}
$$

where $\mathrm{M}$ stands for the effective target mass, $\mathrm{S}$ for the surface area and $\mathrm{L}$ for the distance to the source. Deviations from this simple relation occur if the acceptance for the NEAR detector exceeds the maximum value of $p_{T} \sim 30 \mathrm{MeV}$ from pion decay, because the relative acceptance for neutrinos from pion and $\mathrm{K}_{\mu 3}$ decays is different, and if the geometry of the decay region is not symmetric. All these deviations can be calculated. A measurement at a third distance can be used to check the calculations. The first measurements with two detectors have been performed at CERN by the CDHS [3] and the CHARM [4] Collaborations. Another source of uncertainty occurs in the process of identification of 
interactions of $v_{\mathrm{a}}$. At high energy accelerators beams of $v_{\mu}$ are used and the charged current reaction $(\mathrm{CC})$

$$
v_{\mu} \mathrm{N} \rightarrow \mu^{-} \mathrm{X}
$$

is recorded. The efficiency for detecting this reaction depends on the neutrino energy because of detection thresholds for $E_{X}$ and $E_{\mu}$. The mean energy, however, varies with the distance to the detector because of the decay kinematics. Therefore, the efficiencies of the FAR and NEAR detectors do not cancel completely as suggested in eq(3). The same caution must be taken for the fraction of neutral current (NC) events $v_{\mu} \mathrm{N} \rightarrow v_{\mu} X$ which are misidentified as candidates for the $\mathrm{CC}$ reaction (4). This fraction is, in general, different for the NEAR and the FAR detector.

The sensitivity for establishing a statistically significant deviation of $\mathrm{P}_{\mathrm{aa}}$ from 1 (eq(2)) to detect a term due to $P_{a b} \neq 0$ depends on the event rates in the two detectors and on the remaining uncertainties on deviations from eq(3). I shall come back to some quantitative remarks in connection with my discussion of future projects.

\section{Appearance experiments}

The search for appearance of $v_{b} \neq v_{a}$ in a beam can be performed at a single distance. The ratio L/E gives the limit of sensitivity for $\Delta \mathrm{m}_{\mathrm{ab}}^{2}$. The limit on $\sin ^{2} 2 \theta_{\mathrm{ab}}$ which can be reached depends on the event rate, to some extent also on the neutrino energy because of the threshold for the reaction $v_{\tau} \mathrm{N} \rightarrow \tau \mathrm{X}$ and on the backgrounds both of prompt $v_{\mathrm{b}}$ present in the beam and of other misidentified reactions. For $v_{e}$ this prompt background is about $1 \%$; the prompt $v_{\tau}$ background depends strongly on the proton energy of the accelerator because of the energy dependence of the cross section of the reaction $\mathrm{pN} \rightarrow D_{S} X, D_{S} \rightarrow v_{\tau} \tau$. At the CERN $450 \mathrm{GeV}$ SPS the flux ratio $\varphi\left(v_{\tau}\right) / \varphi\left(v_{\mu}\right) \sim 10^{-7}$ is very favourable for a search for $\nu_{\tau}$ appearance. The dominant limitation is due to, both the event rate (target mass), and to background. Search for $v_{\mu}-v_{\tau}$ oscillation consists in detecting the $v_{\tau}$ induced reaction

$$
v_{\tau} \mathrm{N} \rightarrow \tau \mathrm{X} .
$$

There are several experimental ways to do this. The most direct way is the observation of the $\tau$ decay topology. The transverse decay length is $\sim 90 \mu \mathrm{m}$ and the longitudinal $\sim 250 \mu \mathrm{m}$.

Detection of the decay kink for the dominant decay modes into one charged particle (86\%) or 
of a "star" in the case of $\tau \rightarrow \pi^{-} \pi^{+} \pi^{-}\left(n \pi^{\circ}\right) \vee \tau(14 \%)$ requires a special detector with spacial resolution of better than $10 \mu \mathrm{m}$. Only the emulsion technique gives the required resolution. Another approach is to select events with missing transverse momentum in correlation with the hadron shower direction [5]. This approach, which was not very successful in the search for charm particle production will also be tried.

A third approach is an interesting extension of the disappearance and appearance methods [7]. In $\sim 82 \%$ of all decays the $\tau$ lepton does not produce a muon. Appearance of $v_{\tau}$ therefore reduces the number of events with a muon and increases the number of events without a muon. A measurement of the ratio of muonless events (NC) and of events with a muon (CC) is therefore sensitive to $v_{\tau}$ appearance. Performing another measurement of this ratio in a NEAR detector one can form a double ratio

$$
R=\frac{(N C / C C) F A R}{(N C / C C) \text { NEAR }}
$$

which is less sensitive to deviations of the beam from a point source and from differences in the mean energies in the two detectors. Several such measurements are presently under consideration; I will describe them in Chapter 4.

The search for appearance of $v_{e}$ in a $v_{\mu}$ beam is subject to difficulties from a prompt background of $-1 \%$ which has to be subtracted and from electron/photon discrimination. The background of $v_{e}$ can be measured in a near detector; a double ratio

$$
\mathrm{R}=\frac{(N(e) / C C(\mu)) F A R}{(N(e) / C C(\mu)) N E A R}
$$

therefore can largely eliminate the prompt background problem. The result can also be interpreted as a limit on $v_{\tau}$ appearance, because of the $\sim 17 \%$ branching ratio of $\tau \rightarrow e v_{\mathrm{e}} v_{\tau}$ In all experiments with two detectors a region of sensitivity for $\Delta \mathrm{m}^{2}$ is selected; the lower limit is defined by the FAR detector and the upper limit by the close detector. The upper limit on $\Delta \mathrm{m}^{2}$ is not shown in Table 2 and on figure 11. Appearance experiments, in contrast, are sensitive to oscillation with $\Delta \mathrm{m}^{2} \geq \frac{E}{\mathrm{~L}}$ const. 


\section{Review af results}

No compelling evidence for oscillation has been repoted trom accelerator experiments. There is one new negative result on $v_{\mu}-v_{\tau}$ oscillation reported very recently by the CHAR:M II Collaboration which is approaching in sensitivity the old limit of Ushida et al. [8] obtained with an emuision-hybrid detector (see figure 1) at Fermilab (E531). The CHARM II Collaboration [9]

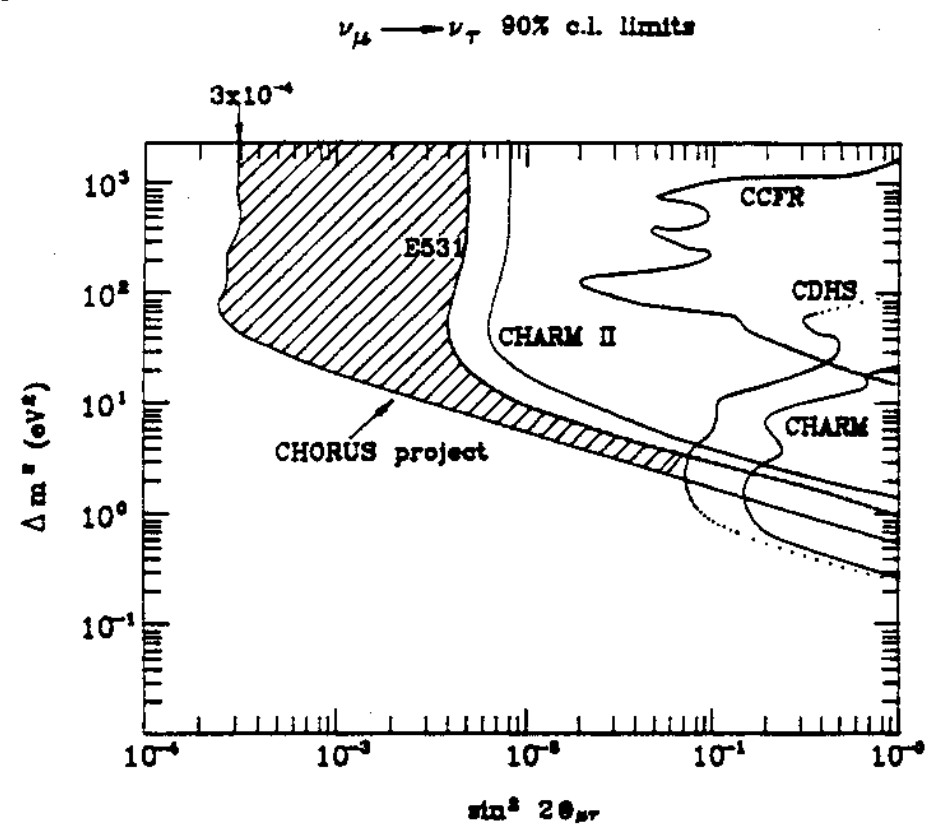

Figure 1 Exclusion plot (90\% C.L.) for $v_{\mu}-v_{\tau}$ oscillation showing results from E531 [8], CHARM II [9], CCFR [10], CDHS and CHARM [11] and the new area which can be explored by the CHORUS [17] and NOMAD [6] experiments.

used a completely different technique. They selected quasi-elastic $v_{\tau}$ interactions followed by the decay $\tau \rightarrow \pi v_{\tau}$. These events appear in the fine-grain calorimeter of the CHARM II detector as a single track followed by a hadronic shower (see figure 2). Background comes from NC reactions with a single charged pion. Requiring a minimum track length of 15 planes of the detector corresponding to $\sim 1.7$ interaction lengths and a shower energy greater than $10 \mathrm{GeV}$ they found 124 events, 77 in the $v_{\mu}$ beam and 47 in the $\bar{v}_{\mu}$ beam. Fitting the kinematical distribution of these events in transverse energy and in the shower energy (figure 3) to a simulation of the $v_{\tau}$ reaction and of the background reaction they derived at the $90 \%$ exclusion region shown in figure 1. The maximum sensitivity is reached for $\Delta \mathrm{m}^{2}=50 \mathrm{eV}^{2}$ where they exclude values of $\sin ^{2} \theta_{\mu \tau}>6.4 \cdot 10^{-3}$. Results of the Fermilab experiment E531 


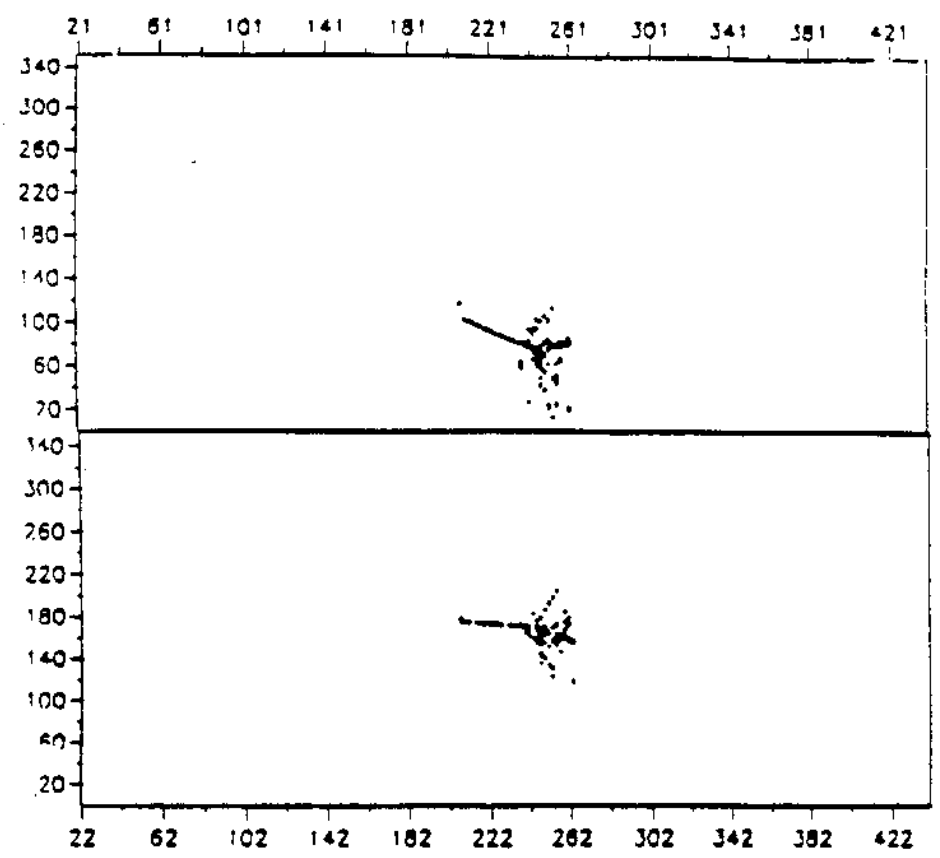

Figure 2 Candidate event of the quasi-elastic $v_{\tau} N \rightarrow\left(\tau \rightarrow \pi v_{\tau}\right) N^{\prime}$ observed by the CHARM II Collaboration [9].

[8], and of searches using the disappearance method of $v_{\tau}$ performed by the CDHS [3] and CHARM [4] Collaborations, as well as those pertormed by the CCFR Collaboration at Fermilab [10], are also displayed in figure 1. The estimated sensitivity of new experiments under preparation at CERN (see Chapter 4) is shown as well for comparison. In conclusion, the excluded region does not yet eliminate the possibility that the dark matter of the universe is formed by $v_{\tau}$. The mixing parameter limit should be further reduced, below the value corresponding to the mixing of the second and third quark family with $\sin ^{2} 2 \theta-10^{-3}$.
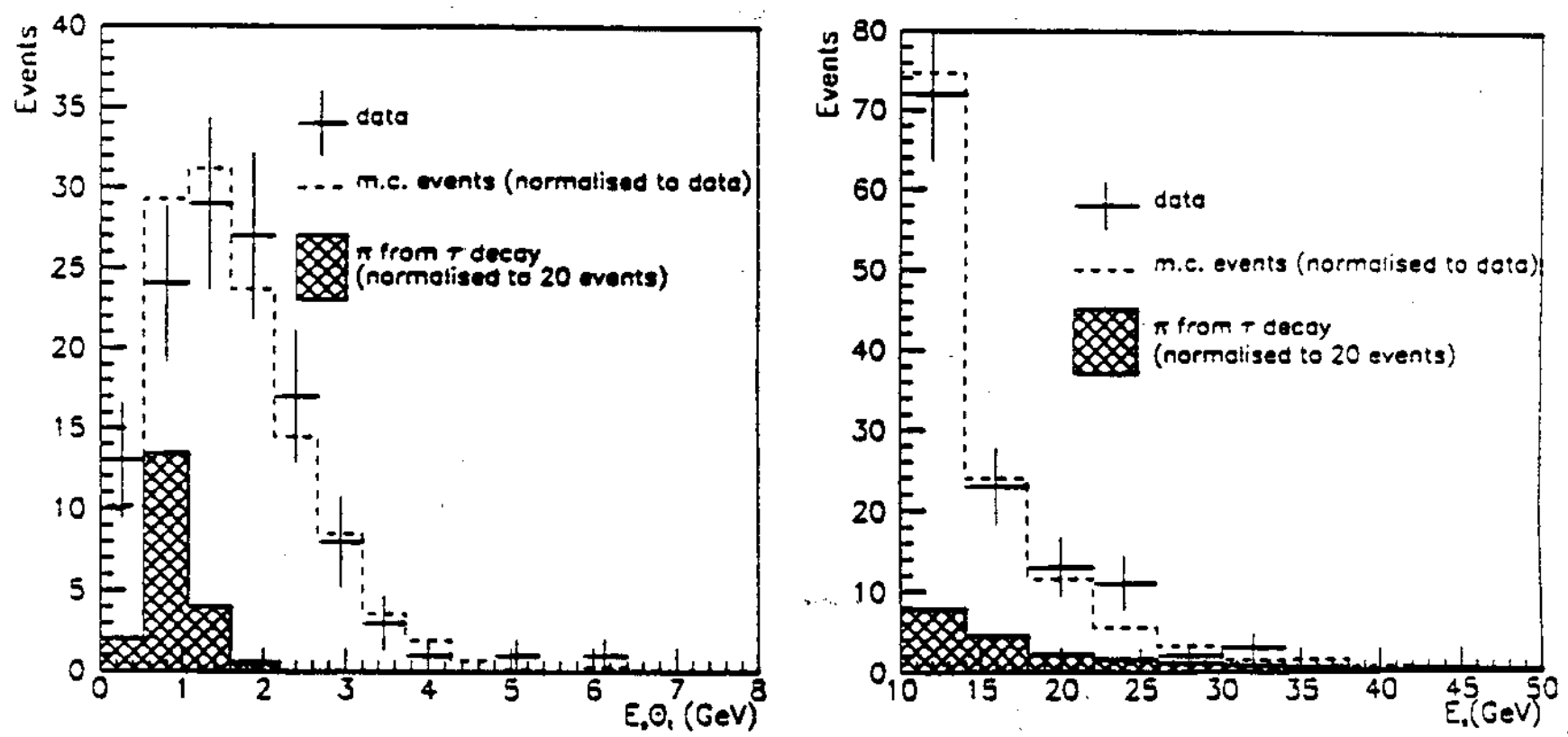

Figure 3 Distribution of (a) $E_{S} \theta_{t}$ and (b) $E_{S}$ for candidates of reaction $v_{\tau} N \rightarrow\left(\tau \rightarrow \pi v_{\tau}\right) N^{\prime}$, CHARM II Collaboration [9] and MC simulation of background. 
much less is known about $v_{e}-v_{\tau}$ mixing. The good news is that we are liberated from the 17 $\mathrm{keV}$ ghost with a $1 \%$ mixing. The other information in this sector is summarized in figure 4 . Assuming again the see-saw mechanism for creating neutrinos masses and that mixing is proportional to the mass difference we must conclude that these experiments are very far away trom detecting an effect.

No new results on $v_{\mu}-v_{e}$ oscillation searches have been reported since the last Neutrino Conference in 1992 [11]. Figure 5 shows a compilation of the present status. The positive effect in PS 191 [12] can now be safely eliminated as it is not confirmed by BEBC [13], CHARM [14], Los Alamos [15] and E776 at BNL [16].

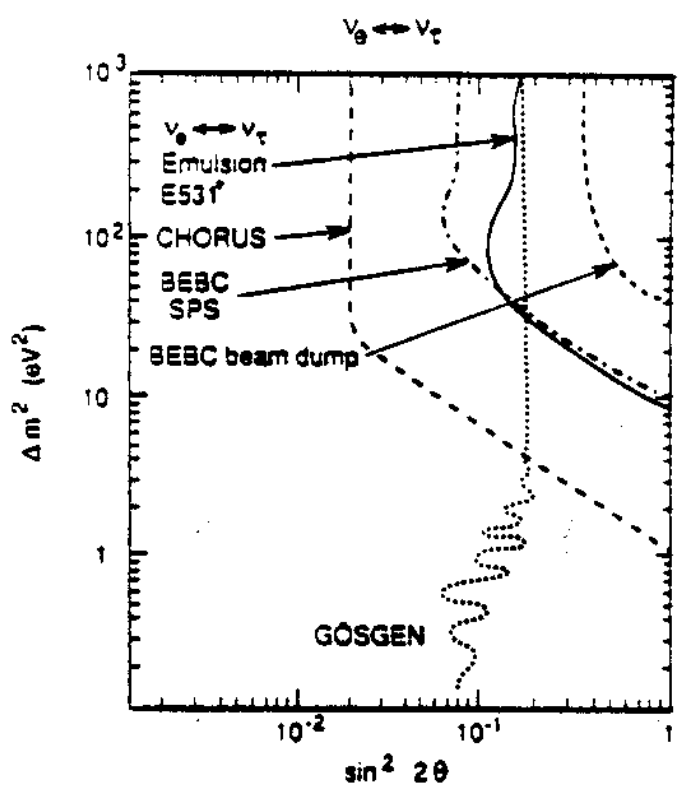

Figure 4 Exclusive plot (90\% C.L.) for $v_{e}-v_{\tau}$ oscillation showing results from E531 [8], BEBC SPS [13] and BEBC Beam Dump [11] and the sensitivity of CHORUS.

\section{New experinents}

Two new experiments searching for $v_{\tau}$ appearance in a $v_{\mu}$ beam are presently under preparation at CERN. CHORUS-WA95 [17] and NOMAD-WA96 [6]. They both aim at detecting the $v_{\tau}$ induced $\mathrm{CC}$ reaction

$$
v_{\tau} \mathrm{N} \rightarrow \tau \mathrm{X}
$$

and several decay modes of the $\tau$. The CHORUS experiment has adopted the emulsion technique to detect the decay topology. In the modern version adopted, events to be 
measured in the emulsion are selected kinematically for the missing transverse momentum feature characteristic for $\tau$ decay. The particle tracks associated with these selected events are measured with scintillating tiber techniques. The detector, therefore, consists of an emuision target and a tracker part (tigure 6) and of a magnet for

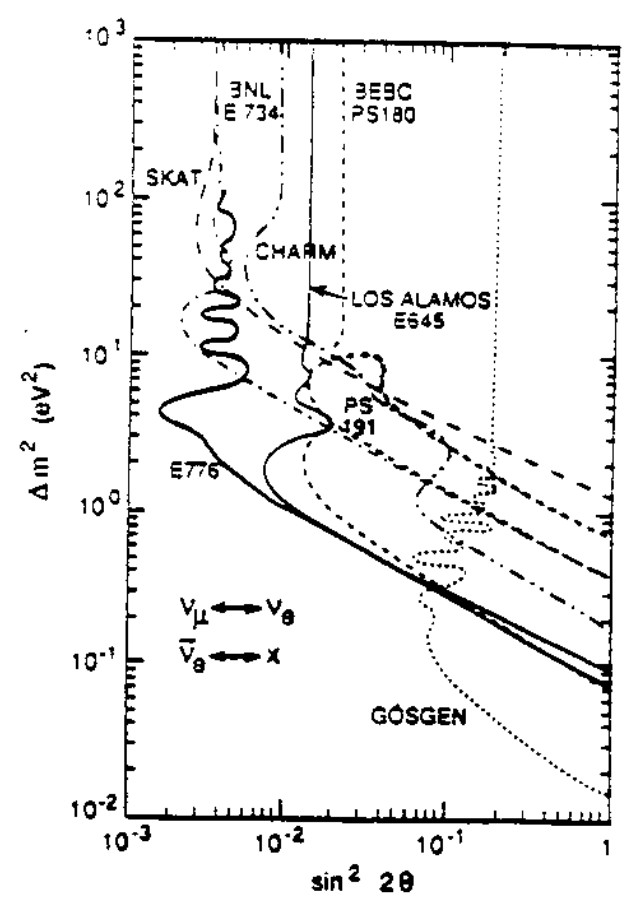

Figure 5 Exclusion plot (90\% C.L.) for $v_{\mu}-v_{\mathrm{e}}$ oscillation showing results from previous experiments [11] and more recent ones from Los Alamos [15], E776 [16], and CHARM [14]. The shaded area. reported [12] as positive evidence is excluded by several experiments.

measuring particle momenta followed by a calorimeter for measuring the hadron shower direction and energy and a muon spectrometer (figure 7). The miscroscopes for emulsion measurements are computer-assisted and measure along the tracks of selected events measured by the scintillating fiber trackers (see figure 6). Using this technique a total efficiency of $\sim 5 \%$ for detecting one of the $\tau$ decay modes can be achieved (Table 1 ). If the $v_{\mu}-v_{\tau}$ oscillation phenomenon should exist at the level of the present $90 \%$ C.L. they would observe 64 events (Table 1) of reaction (6) and a background of $\sim 1.7$ events. If some candidates will be detected their method provides for additional discrimination between reaction (6) and background due to charm particle production by the small $\bar{v}_{\mu}$ and $\bar{v}_{\mathrm{e}}$ components 


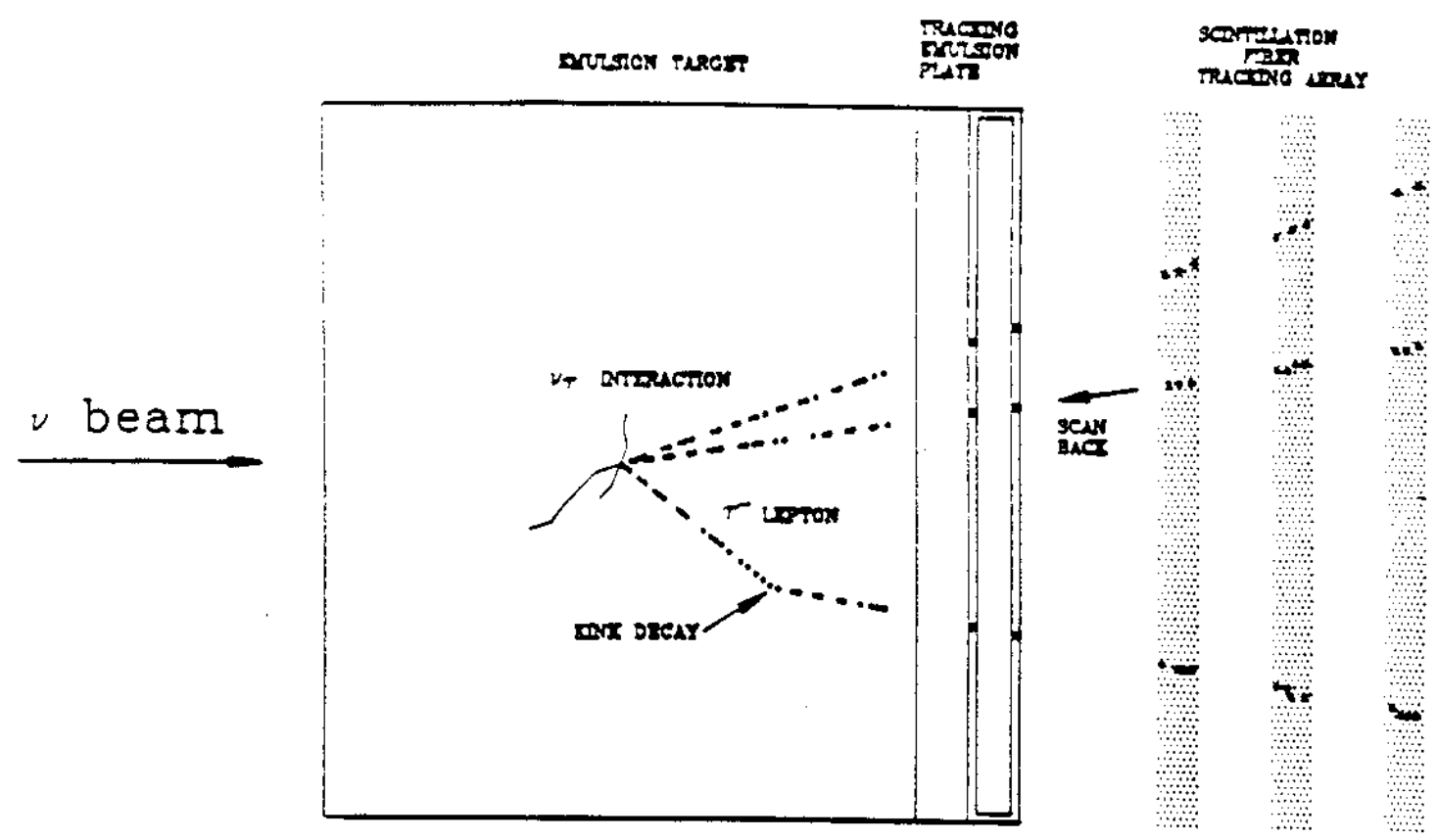

Figure 6 Emulsion target and fiber trackers of the CHORUS experiment [17].

of the beam and due to elastic pion scattering without visible recoil (white kink). The $\tau$ candidate track before the decay kink must balance the $\mathrm{p}_{T}$ of tracks from the hadron vertex (see figure 8, simulated candidate). This is generally not the case for background events. Requiring that vertex configuration in addition

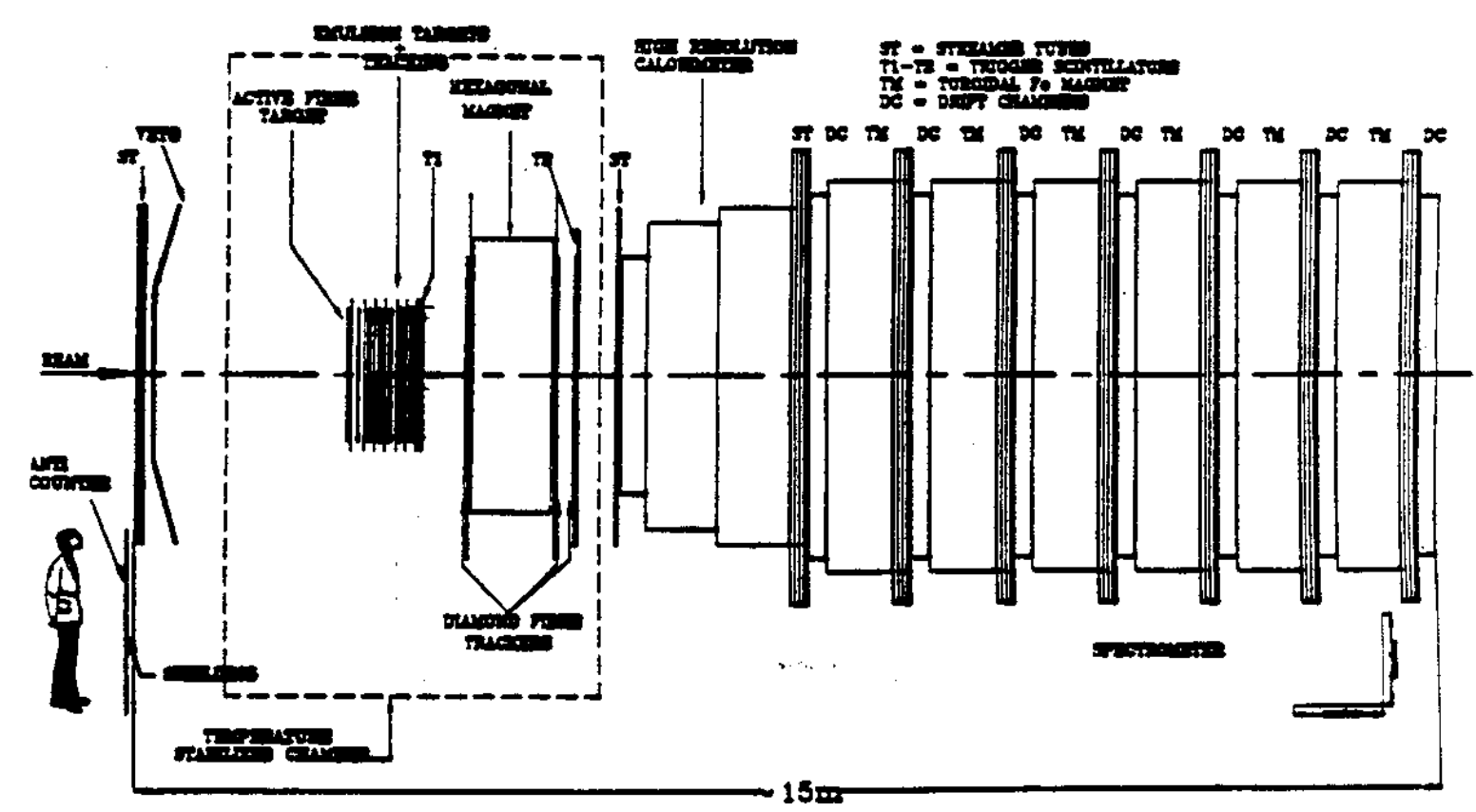

Figure 7 Detector of the CHORUS experiment searching for $v_{\mu}-v_{\tau}$ oscillation [17]. 
reduces the background to 0.4 events and the acceptance efficiency to $4.5 \%$. If no event or one event were observed this would correspond to a $90 \%$ C.L. of $\sin ^{2} 2 \theta_{\mu \tau}>2.7 \cdot 10^{-4}$ at $\Delta \mathrm{m}^{2}$ - $50 \mathrm{eV}$. If some events were observed the discovery potential could be significantly enhanced by using the vertex condition; e.g. tor the observation of events corresponding to $\sin ^{2} 2 \theta \mu \tau \sim 5 \cdot 10^{-4}$ the statistical significance can be increased by a factor of about thirty (see tigure 9).

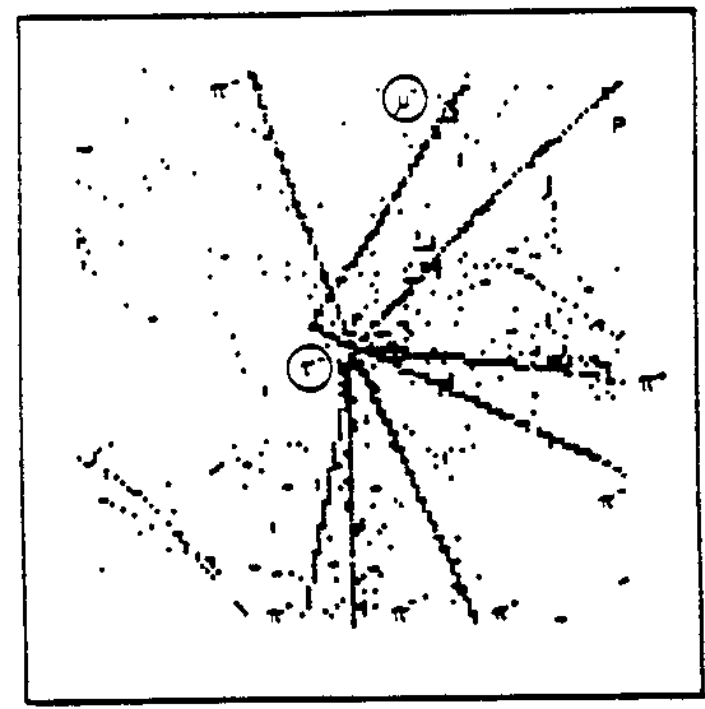

Figure 8 Simulated event $v_{\tau} \mathrm{N} \rightarrow\left(\tau \rightarrow \mu v_{\mu} v_{\tau}\right) \mathrm{X}$ in plane perpendicular to incident neutrino beam [20] showing $\mathrm{p}_{T}$ balancing between the tau track and the hadron tracks.

The NOMAD-WA96 experiment [6] relies entirely on kinematical selection of events. Events due to reaction (6) are expected not to have a $180^{\circ}$ correlation between the charged lepton from $\tau$ decay and the hadron shower direction. The azimuthal correlation between the missing $\mathrm{p}_{T}$ vector $\left(v_{\tau}\right.$ from $\tau$ decay in reaction (6)) and the hadron shower favours large angles. Estimates of the acceptance efficiency, the number of events detected if $\sin ^{2} 2 \theta_{\mu \tau}=$ $5 \cdot 10^{-3}$ and the background are given in Table 1 as well. Because of the larger background, $v_{\tau}$ induced, events observed in this experiment will have smaller statistical significance than in the CHORUS experiment. In the example given before for $\sin ^{2} 2 \theta_{\mu \tau}=5 \cdot 10^{-4}$ (see figure 9) the CHORUS experiment will have a statistical significance which is a factor hundred superior to the NOMAD experiment. The set-up of the NOMAD experiment is shown in figure 10. It is using the magnet of the $\mathrm{UAl}$ experiment for measuring charged particle 
momenta. Electrons are identified by transition radiation. A calorimeter measures the direction and energy of the photon final state. Muons are identified by transmission through an iron absorber.

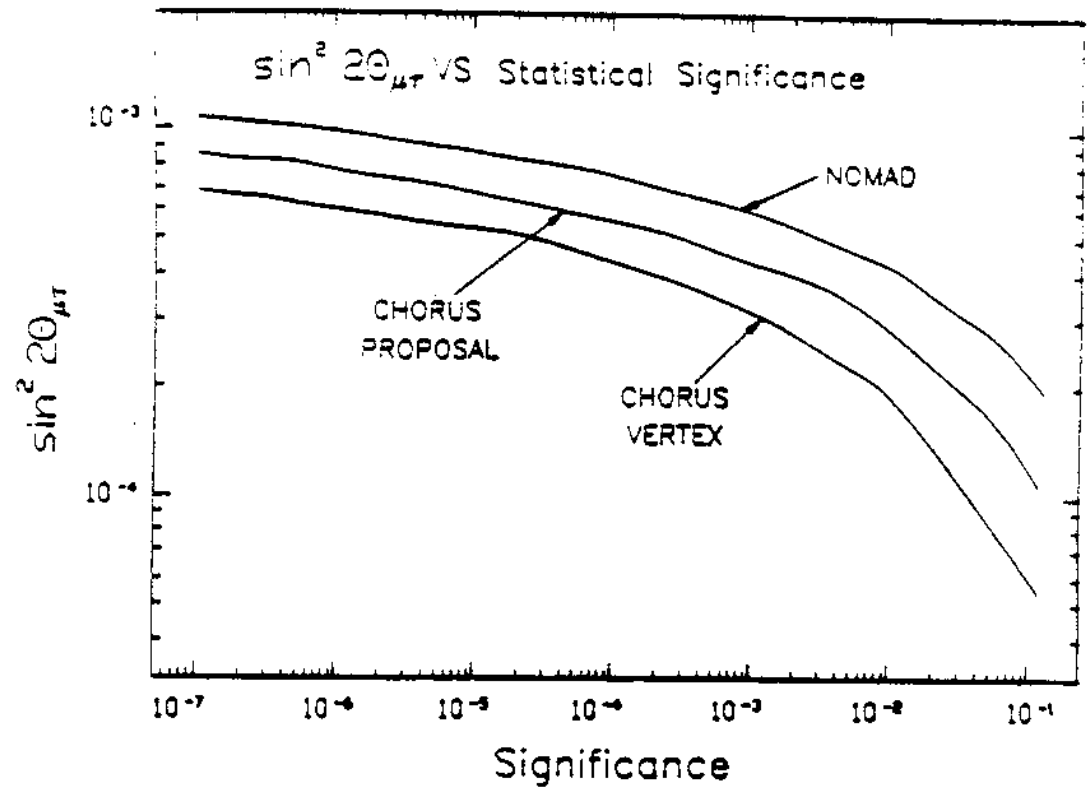

Figure 9 Statistical significance of $v_{\mu}-v_{\tau}$ oscillation events as a function of $\sin ^{2} 2 \theta_{\mu \tau}$ for the NOMAD experiment [6], the CHORUS experiment [17], and CHORUS with the requirement of $\mathrm{P}_{T}$ balance at the vertex [17].

Both experiments are planning data-taking runs from April 1994.

Depending on the outcome, I can anticipate two scenarios :

(1) Some events are found corresponding to $\sin ^{2} 2 \theta_{\mu \tau}>5 \cdot 10^{-4}, \Delta \mathrm{m}^{2}>50 \mathrm{eV}{ }^{2}$.

Then a new experiment will be required which can determine whether the mass of $v_{\tau}$ is of cosmological relevance, i.e. $\mathrm{m}_{\mathrm{v}_{\tau}} \sim 20 \mathrm{eV}$. Such an experiment needs a factor $\sim 20$ larger value of L/E (see eq (1)). This condition can be realized e.g. at the CERN LHC by moving closer to the source and using higher neutrino energy. Because of the higher proton energy ( $7 \mathrm{TeV}$ ) the prompt $v_{\tau}$ background is large and adds to the difficulty of the experiment.

(2) No events above background are found. 
closer to the source and using higher neutrino energy. Because of the higher proton energy ( $7 \mathrm{TeV}$ ) the prompt $v_{\tau}$ background is large and adds to the difficulty of the experiment.

(2) No events above background are found.

A new experiment in the CERN WBB with a sensitivity of $\sin ^{2} 2 \theta_{\mu \tau} \sim 10^{-5}$ would then be interesting. Neither CHORUS nor NOMAD can reach this and a new approach to the detection of the decay topology with a 50 times more massive target is then required. A detector based on the principle of the TPC in cryogenic materials (ICARUS [18]), using $\mathrm{CH}_{4}$, may achieve this sensitivity.

\section{Future prospects}

Apart from persuing the appearance of $v \tau$ with a mass of cosmological relevance it is also interesting to search for smaller neutrino masses. Experimental studies of atmospheric neutrinos seem to indicate the possibility of oscillation $v_{\mu}-v_{\tau}$ or $v_{\mu}-v_{e}$ with $\Delta m^{2} \sim 10^{-3} e^{2}$ and $\sin ^{2} 2 \theta \sim 0.1$. Here a disappearance experiment or an experiment with two detectors combining both disappearance and appearance techniques may be of interest. Several such experiments have been worked out or have been proposed. They are summarized in Table 2. None of them has made a detailed study of systematic uncertainties.

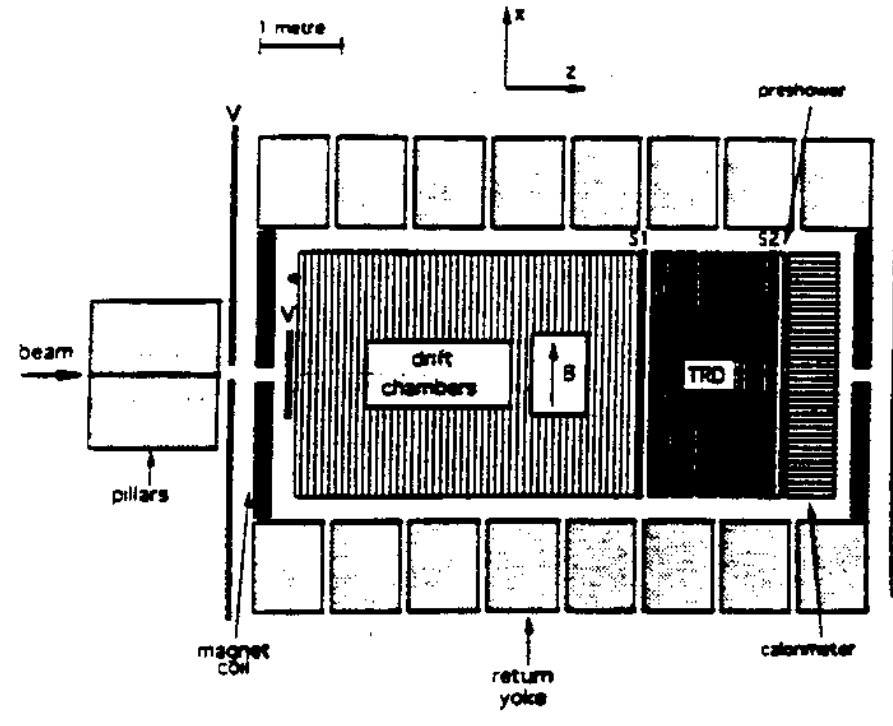

Figure 10 Experimental set-up of the NOMAD experiment [6]. 
In Europe there is the ICARUS experiment with one to three $5 \mathrm{~K}$ tons targets in the Gran Sasso Laboratory using a $v_{\mu}$ beam from CERN and the Superkamiokande experiment with a $20 \mathrm{~K}$ tons target with a $v_{\mu}$ beam trom CERN. The relative merits depend on the possibilities of identification of some classes of events and on the event rate. The distance determines the lower limit of $\Delta \mathrm{m}^{2}$ which can be detected. In the U.S. there are also two projects: the Soudan 2 detector in a $v_{\mu}$ beam from Fermilab and an experiment at BNL using three detectors over $20 \mathrm{~km}$ distance. A comparison of the sensitivities is shown in figures 11 (a) and (b). Having spelt out the difficulties of these experiments in Chapter 2.2 let me also add their advantages
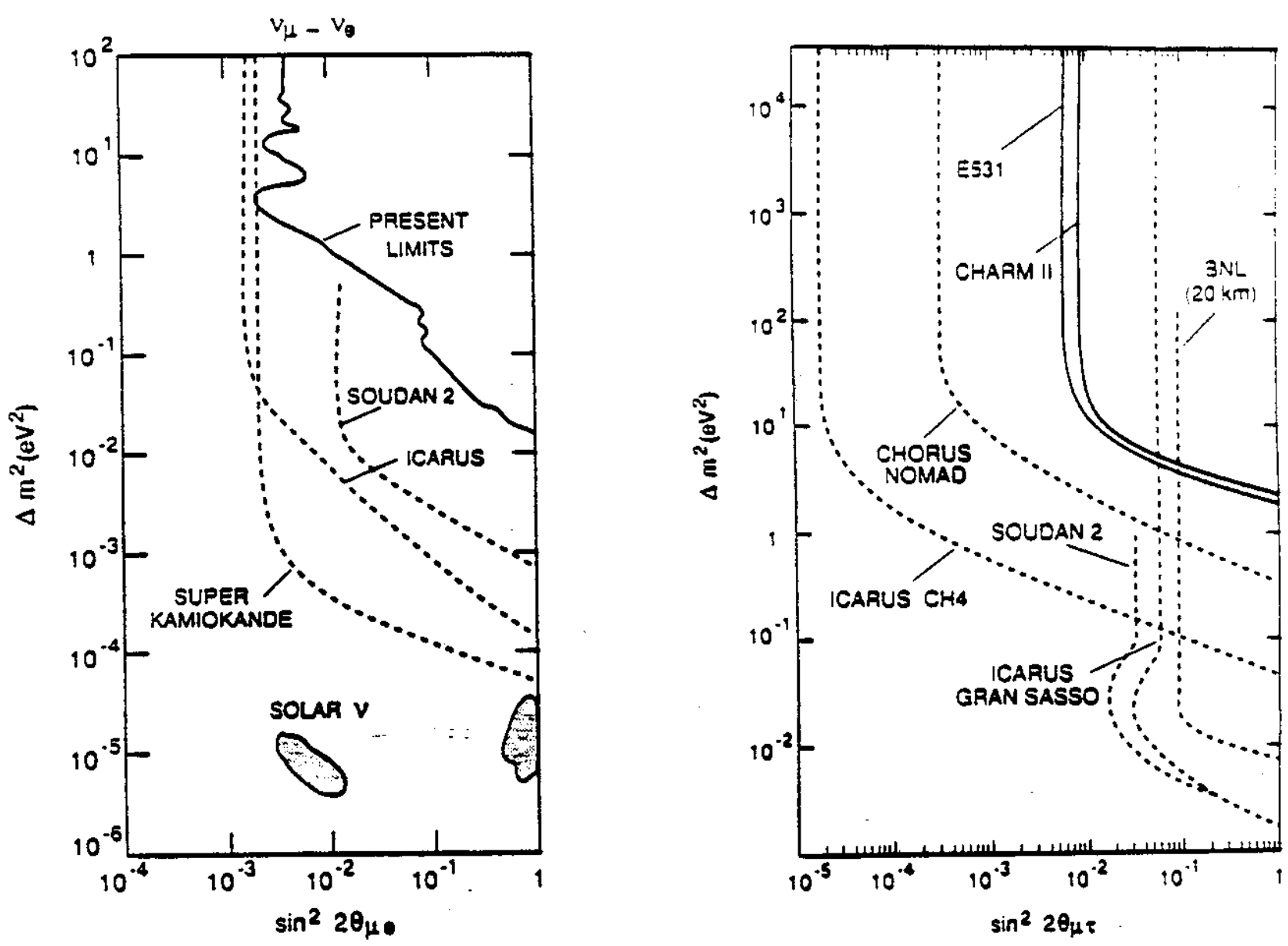

Figure 11 Limits of sensitivity of future projects for oscillation searches. (a) $v_{\mu}-v_{e}$, (b) $v_{\mu}-v_{\tau}$. Limitations of the sensitivity to $\Delta \mathrm{m}^{2}$ because of the NEAR detector measurements are not shown. 
over experiments with atmospheric neutrinos. The spectrum of $E_{V}$ is well known, the beams have known direction and timing, facilitating the rejection of background, and they give high event rate. One can alternate between runs with $v_{\mu}$ and $\bar{v}_{\mu}$ beams and search for matter induced effects which will introduce an asymmetry in the associated event rates. Such matter effects between CERN and Gran Sasso [19] from a comparison of $v_{\mu \rightarrow} v_{e}$ and $\bar{v}_{\mu \rightarrow \bar{v}_{e}}$ oscillation have been studied by Fiorentini and Ricci [19].

\section{Summary}

New accelerator based searches of neutrino oscillation can explore the possibility that the $v_{\tau}$ is the cosmological dark matter candidate. Long baseline experiments are sensitive to another window with $\Delta \mathrm{m}^{2} \sim 10^{-4} \cdot 10^{-3} \mathrm{eV}^{2}$. We are looking forward to their results with a sense of excitement because of the tremendous implications.

\section{Acknowledgements}

In preparing this lecture I have enjoyed discussions with several people, among them $H$. Harari, L. Okun, D. Perkins, C. Rubbia and Y. Totsuka. 
Table 1

Efficiency of $\tau$ - detection

\begin{tabular}{|c|c|c|c|c|c|c|}
\hline Experiment & $\tau-$ Decay mode & $\begin{array}{l}\text { Branching } \\
\text { ratio (BR) }\end{array}$ & $\begin{array}{l}\text { Efficiency } \\
\qquad(\varepsilon)\end{array}$ & $\mathrm{N}_{\tau}{ }^{*}$ & Background & $\begin{array}{l}\text { Background } \\
\text { after vertex } \\
\text { cut }\end{array}$ \\
\hline CHORUS & $\begin{array}{l}\mu^{-} \bar{v}_{\mu} v_{\tau} \\
h^{-}\left(n \pi^{\circ}\right) v_{\tau} \\
\pi^{-} \pi^{+} \pi^{-}\left(n \pi^{\circ}\right) v_{\tau}\end{array}$ & $\begin{array}{l}0.178 \\
0.50 \\
0.138\end{array}$ & $\begin{array}{l}0.098 \\
0.046 \\
0.065\end{array}$ & $\begin{array}{l}23 \\
29 \\
12\end{array}$ & $\begin{array}{l}0.27 \\
0.72 \\
0.71\end{array}$ & \\
\hline & $\varepsilon$ total $=\mathrm{BR} \cdot \varepsilon$ & & 0.0494 & 64 & 1.70 & 0.4 \\
\hline NOMAD & $\begin{array}{l}v_{\tau} c^{-} \bar{v}_{\mu} \\
v_{\tau} \mu^{-}+\bar{v}_{\mu} \\
v_{\tau} \pi^{-} \pi^{-} \pi^{+}+n \pi^{\circ} \\
v_{\tau} \pi^{-} \\
v_{\tau} \rho^{-}\end{array}$ & $\begin{array}{l}0.178 \\
0.178 \\
0.138 \\
0.11 \\
0.23\end{array}$ & $\begin{array}{l}0.135 \\
0.039 \\
0.077 \\
0.014 \\
0.020\end{array}$ & $\begin{array}{r}39 \\
11 \\
18 \\
3 \\
7\end{array}$ & $\begin{array}{r}4.6 \\
2.2 \\
<0.2 \\
<0.2 \\
<0.2\end{array}$ & \\
\hline & $\varepsilon$ total & . & 0.0477 & 78 & 6.8 & 6.8 \\
\hline
\end{tabular}

* The number of events corresponds to $\sin ^{2} 2 \theta=5 \cdot 10^{-3}$ and $\Delta m^{2} \geq 40 \mathrm{eV}^{2}$ and a run of $2.4 \cdot 10^{19}$ protons on target. 
Table 2

Long baseline neutrino oscillation projects

\begin{tabular}{|l|r|r|r|r|}
\hline \multicolumn{1}{|c|}{ Detector } & Distance & Mass & $\left.\Delta \mathrm{m}^{2} \mu \mathrm{e}^{*}\right)$ & $\left.\Delta \mathrm{m}^{2} \mu \tau^{*}\right)$ \\
\hline $\begin{array}{l}\text { ICARUS (TPC) } \\
\text { SUPERKAMIO- } \\
\text { KANDE (water) }\end{array}$ & $731 \mathrm{Km}$ & $5 \mathrm{~K}$ tons & $2 \cdot 10^{-4}$ & $2 \cdot 10^{-3}$ \\
$\begin{array}{l}\text { SOUDAN 2 (calorim.) } \\
\text { (P822) }\end{array}$ & $730 \mathrm{Km}$ & $1+5 \mathrm{~K}$ tons & $10^{-3}$ & $5 \cdot 10^{-5}$ \\
BNL 889 (water) & $20 \mathrm{Km}$ & $6 \mathrm{~K}$ tons & $\mathrm{K}$ tons & $2 \cdot 10^{-3}$ \\
\hline
\end{tabular}

*) Limit for $\sin ^{2} 2 \theta=1$ 


\section{Reterences}

[1] K. Lande. Proc. Neutrino 92, Nucl, Phys.B(Proc. Supplu31(1993)

K. Nakamura. Kamiokande Coll., loc.cit p. 105

O.L. Anosov et al. SAGE Coll., loc.cit. p. 111

P. Anselmann et al.. GALLEX Coll., leceit. p. 117

[2] H. Harari, Phys, Lett. 216B(1989)413

[3] CDHS Collab.. F. Dydak et al., Phys._Lett. 134B(1984)281

[4] CHARM Collab., F. Bergsma et al.. Phys. Lell. 142B(1984)103

[5] C. Albright et al.. Phys, Lett. 84B(1979)123

[6] L. DiLella, Proc. Neutrino 92, Nucl. Phys. B(Proc. Suppl.13 I(1993)319

[7] K. Winter, Int. Workshop on Neutrino Telescopes, Venice 1988, Proc. edited by M. Baido Ceolin, p. 184.

K. Winter, Proc. Arkansas Gamma-Ray and Neutrino Workshop 1989, Nucl. Phys. B (Proc Suppl) 14A(1990)321

[8] N. Ushida et al., E531 Collab., Phys.Rev.Lett. 57(1986)2897

[9] CHARM II Collab., M. Gruwé et al., Phys. Lett. B (in print)

[10] CCFR Collab., C. Habes et al., Phys. Rev, Lett. 52(1984)1384

[11] J. Schneps, Invited talk, Neutrino 92, Nucl. Phys. B (Proc. Suppl) 31(1993)307

[12] G. Bernardi et al., Bhys, Lett. 181B(1986)173

[13] BEBC. C. Angelini et al., Phys. Lett. 179B(1986)307

[14] CHARM Collab., F. Bergsma et al., Zf.Phys, C40(1988)171

[15] LOS ALAMOS, T: Dombeck et al., Phys. Lett.

[16] E776(BNL) L.A. Ahrluder et al., Phys. Rey. D31(1985)2732

[17] CHORUS Collab., N. Armenise et al., CERN-SPSC/90-42,SPSC P254 and PPE int report 1993.

[18] C. Rubbia, CERN-PPE/93-08 
[19] Fiorentini, and Ricci, Int. Workshop on Neutrino Telescopes, Venice 1993 (to be published)

[20] K. Winter, Acta Physica Hungarica 68(1990) 135 